\title{
Detection of activating mutations in liquid biopsy of Egyptian breast cancer patients using targeted next-generation sequencing: a pilot study
}

\author{
Neemat Kassem ${ }^{1}$, Hebatallah Kassem ${ }^{1 *}$, Loay Kassem ${ }^{2}$ and Mohamed Hassan²
}

\begin{abstract}
Background: Breast cancer $(\mathrm{BC})$ is the $2^{\text {nd }}$ most prevalent malignancy worldwide and is the most prevalent cancer among Egyptian women. The number of newly described cancer-associated genes has grown exponentially since the emergence of next-generation sequencing (NGS) technology. We aim to identify activating mutations in liquid biopsy of Egyptian breast cancer patients using targeted NGS technology. We also demonstrate the microsatellite instability (MSI) status using BAT25, BAT26, and NR27 markers which are tested on the Bioanalyzer 2100 system.

Results: Twenty-one variants were detected in 15 genes: 7 Substitution-Missense, 12 Substitution-coding silent, and 2 Substitution-intronic. Regarding ClinVar database, out of 21 variants there were 14 benign variants, 3 variants with conflicting interpretations of pathogenicity, 3 variants not reported, and 1 drug response variant. TP53 p.(Pro72Arg) missense mutations were found in 75\% of patients. PIK3CA p.(lle391Met), KDR p.(Gln472His) missense mutations were detected in 25\% of patients each. Two patients revealed APC gene missense mutation with p.(Ile1307Lys) and p.(Glu1317Gln) variants. Only one patient showed ATM p.(Phe858Leu) gene mutation and one showed FGFR3 p.(Ala719Thr) variant. Regarding microsatellite instability (MSI) status, 2/8 (25\%) patients were MSS, 3/8 (37.5\%) patients were MSI-L, and 3/8 (37.5\%) patients were MSI-HI.

Conclusion: It is essential to use and validate minimally invasive liquid biopsy for activating mutations detection by next-generation sequencing especially in patients with inoperable disease or bone metastasis. This work should be extended with larger patient series with comparison of genetic mutations in liquid-based versus tissue-based biopsy and longer follow up period.
\end{abstract}

Keywords: Breast cancer, Next-generation sequencing, Activating mutations, Microsatellite instability

\section{Background}

Breast cancer $(\mathrm{BC})$ is the $2^{\text {nd }}$ most prevalent and lethal malignancy worldwide and is the most prevalent cancer among Egyptian women [1]. In Egypt, National Cancer Registry Program (NCRP) revealed that the commonest cancer sites were liver, breast, and bladder $(23.8 \%, 15.4$

\footnotetext{
* Correspondence: Heba.kasem@hotmail.com

'Clinical and Chemical Pathology Department, Kasr Al Ainy Centre of Clinical Oncology \& Nuclear Medicine, School of Medicine, Cairo University, Cairo, Egypt

Full list of author information is available at the end of the article
}

and $6.9 \%$, respectively) in both genders; liver and bladder (33.6\% and $10.7 \%$, respectively) in men; and breast and liver (32\% and $13.5 \%$, respectively) in females [2]. Bone is the most common location of $\mathrm{BC}$ metastasis; these metastases are found in $65-75 \%$ of metastatic BC patients [3]. Furthermore, bone has been noted to be the most common location of first distant BC relapse [4].

Decades of research has generated the recognition that cancer is a genetic disorder, revealing that it is the accumulation of molecular alternations which is the principle factor of tumorigenesis, guiding the acquisition of the 
malignant phenotype [5]. The number of newly described cancer-associated genes has grown exponentially since the emergence of next-generation sequencing (NGS) technology [6].

For many decades, the single available substance for molecular testing was the patient's formalin-fixed paraffinembedded (FFPE) tumor tissue. This FFPE samples have many advantages as it is accessible material, simple for usage and storage. Also, it gives the chance to choose appropriate tumor tissue, elevating the sensitivity of genetic mutation detection assays [7]. On the other hand, FFPE material has obvious disadvantages, such as inability to acquire in cases of inoperable tumors and bone metastases with some difficulties to capture the tumor's heterogeneity. Moreover, this genetic material collected as a result of paraffin processing of tissue, is commonly of low/poor quality, and not adequate for molecular profiling [8]. The most essential point that the molecular profile of cancer is transformed, basically after targeted therapy and these alternations cannot be noticed by testing the 1ry tumor material but require invasive tissue rebiopsies [9].

The presence of neoplastic features in the plasma DNA of cancer patients was first observed in 1989 [10]. Later on, several reports have found that testing of cell-free tumorderivative nucleic acids in cancer patient's body fluids (serum, plasma, urine, stool, bronchoalveolar lavage, etc.) may be used to determine tumor specific variations [11]. The word liquid biopsy has came up demonstrating the use of those minimally invasive materials for tumor characterization. The mutations noticed in liquid biopsies reflect mutations found in the patient's tumor itself. As well as, circulating tumor nucleic acids (ctNA) analysis could eventually determine more genetic alternations compared to analysis of a particular area in a FFPE tumor tissue, as it arises from the whole tumor's area and/or metastasis present in the patient's body, so being characteristic of intra and inter-tumor heterogeneity [12]. The usage of plasma samples for ctNA analysis has recently become attainable because of the improvement of sensitive molecular techniques that can determine with high accuracy minimal amounts of ctNAs that are present in these liquid biopsies. For this intend, several techniques have been used, such as digital PCR, real-time PCR, Arms PCR, and NGS [13].

In this study, we aim to identify activating mutations in liquid biopsy of Egyptian breast cancer patients using targeted next-generation sequencing technology. We also demonstrate the microsatellite instability (MSI) status using BAT25, BAT26, and NR27 markers which are tested on the Bioanalyzer 2100 system.

\section{Methods}

\section{Study population}

The study included 8 Egyptian breast cancer patients who are attending to outpatient clinic of our department. Patients were selected according to the following inclusion criteria: adult, females, and confirmed pathological examination of invasive carcinoma of no specific type. Seven patients received neoadjuvant chemotherapy and underwent surgical interventions. After that, each patient was treated individually and received either adjuvant chemotherapy or hormonal treatment according to international guidelines. At this stage, blood samples were withdrawn from those patients under strict sterile conditions for molecular testing. Clinico-pathological features at diagnosis were collected from patients' records. This study was done in the molecular Lab. of our institution, in the period from December 2019 to June 2020. The study was approved by Institutional Review Board (IRB)-11-2019 of our clinical oncology department. All procedures carried out in the study including human participants were in agreement with the ethical standards of the institutional research committee and with the 1964 Helsinki declaration and its later amendments (GCP guidelines) or comparable ethical standards.

\section{DNA extraction}

According to the manufacturer protocol, genomic DNA (gDNA) was extracted from those liquid biopsies with the QIAamp DNA Mini kit (QIAGEN, Germany, Cat No./ID: 51304) and was eluted in $60 \mu \mathrm{L}$ volume. Concentration of extracted DNA samples was measured by the Qubit dsDNA High Sensitivity (HS) assay kit (Life Technologies-Fisher Scientific, Cat No.:Q32851). Testing quality and amplifiability of the extracted gDNA samples was done by quantitative PCR (qPCR) technology. All samples with $\Delta$ Cq value below 5 can be selected for further use.

\section{Library preparation and sequencing}

Following the manufacturer instructions, the libraries were prepared using AmpliSeq ${ }^{\text {TM }}$ for Illumina Cancer Hotspot Panel v2 (Illumina, Inc., US, Cat. No.: 20019161) which is a targeted next generation sequencing assay detecting actionable mutations across the hotspot regions of 50 genes. Quality of the libraries was checked out by Agilent 2100 Bioanalyzer device utilizing the DNA 1000 reagents and Chips (Agilent Technologies, Santa Clara, California, Cat. Code: 5067-1504). The expected PCR product is $186-277 \mathrm{bp}$, which indicates successful library amplification. Patients' libraries together with PhiX control library were normalized and equal volumes were pooled to form the terminal sequencing library. The AmpliSeq ${ }^{\mathrm{m}}$ for Illumina Cancer Hotspot Panel achieves detection limits of 5\% variant allele frequency across 207 amplicons with $>95 \%$ of bases covered at $\geq 500 \times$ [14]. Sequencing was done using Cancer Hotspot Panel v2 Nano kit on MiSeqDx device (llumina) 
with a $2 \times 150$ bp read length and total time of $\sim 17 \mathrm{~h}$ which involve cluster generation, sequencing, and base calling on the MiSeqDx system.

\section{Bioinformatics and data analysis}

Bioinformatics and data analysis start from checking each run quality through assessing the specifications based on Illumina PhiX control library which support cluster densities between $865-965 \mathrm{k} / \mathrm{mm}^{2}$ clusters passing filter for $\mathrm{v} 2$ chemistry. The second item is the quality score (Q-score) which is a prediction of the probability of an error in base calling. The percentage of bases > Q30 is averaged across the entire run. The quality scores for v2 chemistry $>80 \%$ bases higher than Q30 at $2 \times 150$ bp. The assembly of the reads was run to Genome Reference Consortium Human Build 37 (GRCh37) which is the human reference genome (version hg19). Image processing and Variant Call Format (VCF) file generation were further analyzed, we then annotated these variants using Illumina variant interpreter. Each variant is linked to numerical identifier in Catalogue of Somatic Mutations in Cancer (COSMIC) database. The likely impact of amino acid changes was determined with In Silico Predictions (Sift \& PolyPhen) and Functional Analysis through Hidden Markov Models (v2.3) (FATHMM) prediction. The variants were categorized as benign or pathogenic according to ClinVar database. Mutations with low depth, which indicate $\leq 50 \times$ depths, mutations with $\leq 5 \%$ variant allele frequency, variants quality if $<$ $80 \%$ and finally, variant that did not found in COSMIC database were filtered out.

\section{Microsatellite instability analysis}

We assessed the microsatellite instability (MSI) status using 3 primer sequences (BAT25, BAT26, and NR27) according to manufacturer instructions with PCR products were analyzed on Agilent 2100 Bioanalyzer system as previously described. Tumor DNA was compared to that of healthy control with peaks present in the tumor that were not found in the normal subjects indicated instability of a marker. Patients with no varied markers were considered as microsatellite stable (MSS). Patients with only one varied microsatellite marker were considered as microsatellite instability-low (MSI-L) and those with $\geq 2$ varied markers were classified as microsatellite instability-high (MSI-HI) [15].

\section{Results}

\section{Patients' characteristics}

Eight breast cancer female patients were enrolled in the study and their clinico-pathological features at diagnosis including stages, hormonal receptor status, and molecular subtypes were shown in Table 1. Only one patient had metastatic BC at first presentation and she was 45
Table 1 Characteristics of breast cancer patients

\begin{tabular}{|c|c|}
\hline Patient's characteristics & $\begin{array}{l}\text { Total No. }=8 \\
\text { No. }(\%)\end{array}$ \\
\hline \multicolumn{2}{|l|}{ Age (years) } \\
\hline Mean \pm SD & $40 \pm 7.01$ \\
\hline Median & 41 \\
\hline Range & $29-48$ \\
\hline \multicolumn{2}{|l|}{ Stage at diagnosis } \\
\hline$\|$ & $2 / 8(25 \%)$ \\
\hline III & $5 / 8(62.5 \%)$ \\
\hline IV & $1 / 8(12.5 \%)$ \\
\hline \multicolumn{2}{|l|}{$\begin{array}{l}\text { Immunohistochemistry } \\
\text { ER status }\end{array}$} \\
\hline Positive & $5 / 8(62.5 \%)$ \\
\hline Negative & $3 / 8(37.5 \%)$ \\
\hline \multicolumn{2}{|l|}{ PR status } \\
\hline Positive & 4/8 (50\%) \\
\hline Negative & $4 / 8(50 \%)$ \\
\hline \multicolumn{2}{|l|}{ HER2 neu } \\
\hline Positive & $1 / 8(12.5 \%)$ \\
\hline Negative & $7 / 8(87.5 \%)$ \\
\hline \multicolumn{2}{|l|}{ KI 67} \\
\hline High & $4 / 8(50 \%)$ \\
\hline Low & $1 / 8(12.5 \%)$ \\
\hline Unknown & $3 / 8(37.5 \%)$ \\
\hline \multicolumn{2}{|l|}{ Molecular subtype } \\
\hline Luminal A & $1 / 8(12.5 \%)$ \\
\hline Luminal B & $4 / 8(50 \%)$ \\
\hline Triple negative disease & $3 / 8(37.5 \%)$ \\
\hline \multicolumn{2}{|l|}{ Metastasis } \\
\hline Metastatic: & $6 / 8(75 \%)$ \\
\hline Liver & $4 / 6(66.7 \%)$ \\
\hline Bone & $3 / 6(50 \%)$ \\
\hline Lung & $4 / 6(66.7 \%)$ \\
\hline Brain & 0 \\
\hline Local recurrence & $1 / 6(16.7 \%)$ \\
\hline Early disease, non metastatic: & $2 / 8(25 \%)$ \\
\hline \multicolumn{2}{|l|}{ Microsatellite instability (MSI) status } \\
\hline Microsatellite stable (MSS) & $2 / 8(25 \%)$ \\
\hline Microsatellite instability-low (MSI-L) & $3 / 8(37.5 \%)$ \\
\hline Microsatellite instability-high (MSI-HI) & $3 / 8(37.5 \%)$ \\
\hline
\end{tabular}

years old, 5 patients were proven to be metastatic $\mathrm{BC}$ during therapy and 2 patients had early non-metastatic $\mathrm{BC}$. The histological subtype of all patients was invasive carcinoma of no specific type with median age of 41 years (range, $29-48$ years). 


\section{Activating mutations' results}

Regarding mutational analysis, the variant allele frequency (VAF) was used to differentiate germline from somatic variants. A germline variant is identified with a $50 \%$ (heterozygous) or $100 \%$ (homozygous) VAF. Acquired variant is present with a lower VAF as it is not found in all cells. Other factors may also contribute to VAF such as technical issues (polymerase chain reaction/amplification bias) can skew VAF. Also, somatic mutations may occur with VAF of $50 \%$ if the number of malignant cells in the analyzed sample is high. Finally, genetic features affect the VAF. VAF for each identified variant in each patient is shown in Table 2.

Initial filtering yielded 42 variants as shown in Table 3 . By searching about these variants in the COSMIC database version 92, we found that 13 variants did not found in COSMIC database, 6 variants are classified as a noncoding variant in COSMIC, because it was annotated in the intron of a transcript and 2 variants have been found as SNP. As a result, these variants were eliminated from our further discussion. The remaining 21 variants showed different activating mutations: 7 substitutionmissense, 12 substitution-coding silent, and 2 substitution-intronic as shown in Fig. 1. These 21 activating mutations were found in 15 genes. Regarding ClinVar database, out of 21 variants there were 14 benign variants, 3 variants with conflicting interpretations of pathogenicity, 3 variants not reported, and 1 drug response variant.

\section{Substitution-missense mutations}

Tumor protein TP53 (TP53) gene mutations were substitution-missense mutations and were detected in 6 patients. All were p.(Pro72Arg) drug response variant which occur as a result of substitution at c.215C>G. Phosphatidylinositol 4,5-bisphosphate 3-kinase catalytic subunit alpha (PIK3CA) gene mutation was detected in 2 patients, both were substitution-missense mutations p.(Ile391Met) as a result of substitution at c.1173A $>$ G and classified as benign variant in ClinVar database. Kinase insert domain receptor $(K D R)$ gene is a vascular endothelial growth factor receptor 2 (VEGFR2) gene and its mutation was found in 2 patients. They were substitution-missense mutations p.(Gln472His) that result from substitution at c.1416A $>\mathrm{T}$ and such variant not reported in ClinVar database. Only one patient revealed ataxia telangiectasia mutated $(A T M)$ gene mutation. It was substitution-missense mutation p.(Phe858Leu) resulting from substitution at c. $2572 \mathrm{~T}>\mathrm{C}$ and reported in ClinVar database as a variant of conflicting interpretations of pathogenicity. However, it was predicted to be pathogenic variant according to FATHMM prediction score. One patient showed substitution-missense mutation in FGFR3 gene (c.2155G>A). It is reported in
ClinVar database as likely benign variant, but it is pathogenic at FATHMM prediction. Two patients showed substitution-missense mutations in APC Regulator of WNT signaling pathway $(A P C)$ gene. One patient showed p.(Ile1307Lys) variant as a result of substitution at c.3920T>A and classified in ClinVar database as a variant of conflicting interpretations of pathogenicity, risk factor. The other patient was p.(Glu1317Gln) resulting from substitution at c.3949G $>\mathrm{C}$ and considered in ClinVar database as a variant of conflicting interpretations of pathogenicity. These $2 A P C$ variants were predicted to be pathogenic variants according to FATHMM prediction score.

\section{Substitution-coding silent mutations}

Seven patients revealed substitution-coding silent mutations in $A P C$ gene which were benign p.(Thr1493=) variant resulting from substitution at c.4479G $>$ A. Two benign substitution-coding silent mutations were detected in Ret Proto-Oncogene (RET) gene. The first one was p.(Ser904=) variant as a result of c. $2712 \mathrm{C}>\mathrm{G}$ and was found in 2 patients. The other one was p.(Leu769=) resulting from c.2307G $>\mathrm{T}$ and was detected in 8 patients. Again substitution-coding silent mutations were detected in isocitrate dehydrogenase $(\mathrm{NADP}(+)) \quad 1$ (IDH1) gene in only 1 sample, HRas Proto-Oncogene, GTPase (HRAS) gene in 4 samples, epidermal growth factor receptor $(E G F R)$ gene in 6 samples, MET ProtoOncogene, receptor tyrosine kinase $(M E T)$ gene in only 1 sample, fibroblast growth factor receptor 3 (FGFR3) gene in 8 samples, and Serine/threonine kinase 11 (STK11) gene in only 1 sample. These variants were p.(Gly105=), p.(His27=), p.(Gln787=), p.(Ile377=), p.(Thr653=), and p.(Tyr272=), respectively resulting from substitution at c.315C $>\mathrm{T}$, c. $81 \mathrm{~T}>\mathrm{C}$, c. $2361 \mathrm{G}>\mathrm{A}$, c. $1131 \mathrm{C}>\mathrm{T}$, c. $1959 \mathrm{G}>\mathrm{A}$, and c.816C $>\mathrm{T}$, respectively. Platelet-derived growth factor receptor A (PDGFRA) gene revealed 3 substitution-coding silent mutations p.(Val824=), p.(Pro577=), and p.(Pro567=) that result from c.2472C $>$ T, c. $1731 \mathrm{G}>\mathrm{A}$ and $\quad$. $1701 \mathrm{~A}>\mathrm{G}$, respectively.

\section{Substitution-intronic mutations}

Finally, 2 substitution-intronic were found in Fms related receptor tyrosine kinase 3 (FLT3) gene in 7 samples and SWI/SNF related, matrix associated, actin dependent regulator of chromatin, subfamily $\mathrm{B}$, member 1 (SMAR CB1) gene in only 1 sample as a result of substitution at c.1310-3T>C and c.1119-41G >A, respectively.

\section{Microsatellite instability status}

Regarding microsatellite instability (MSI) status, 2/8 (25\%) patients were MSS, 3/8 (37.5\%) patients were MSI-L, and $3 / 8$ (37.5\%) patients were MSI-HI. 


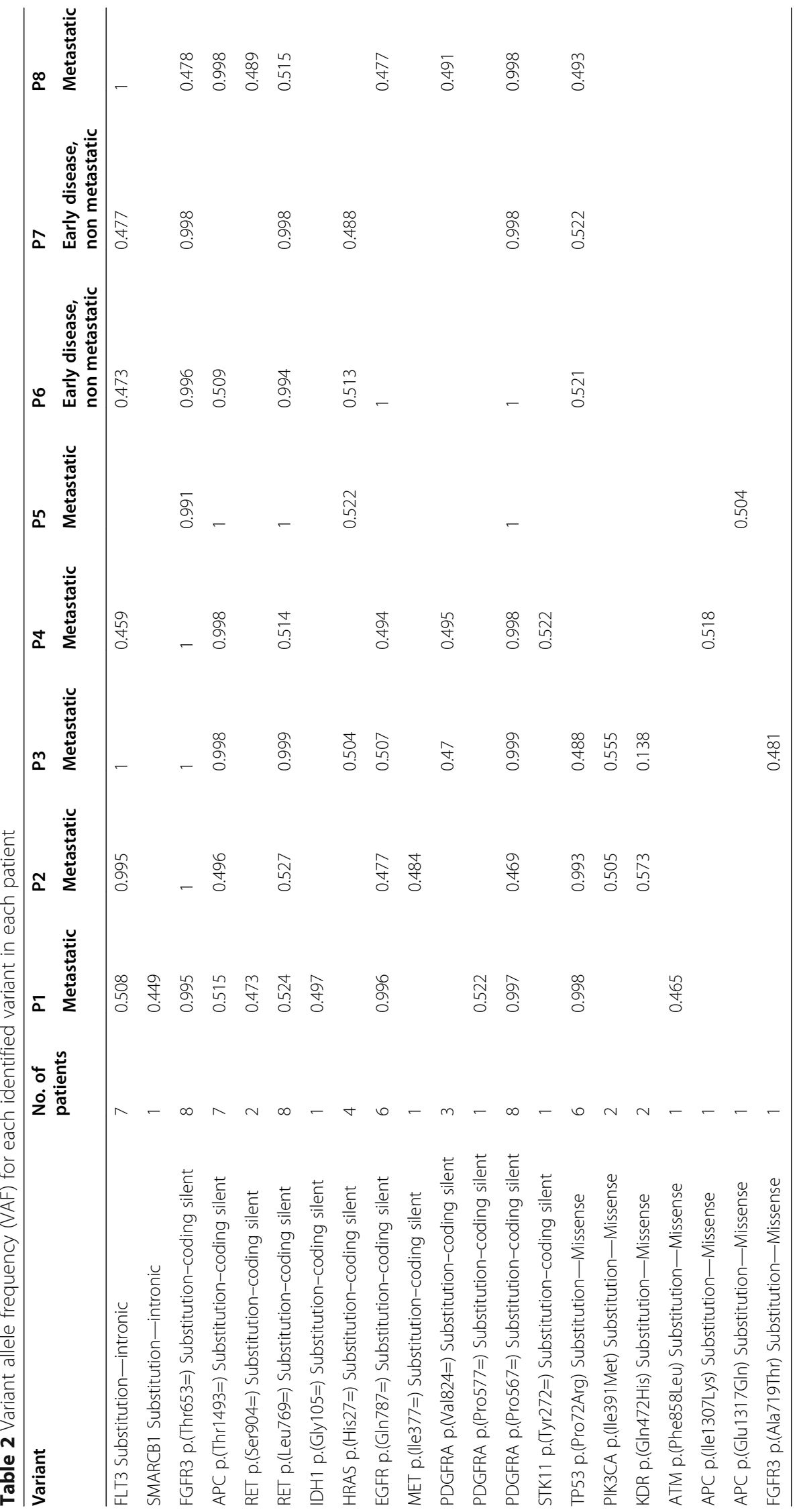




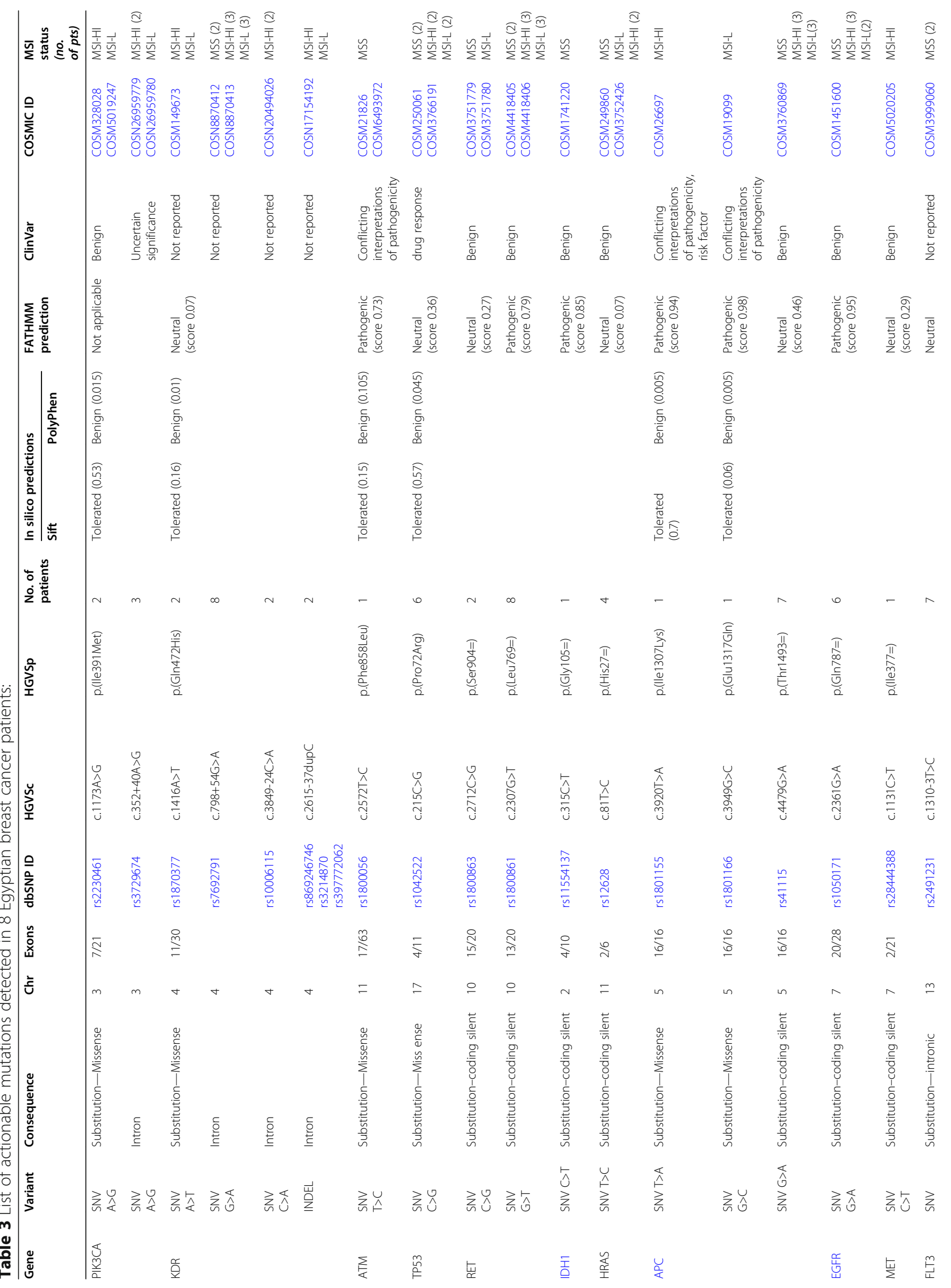




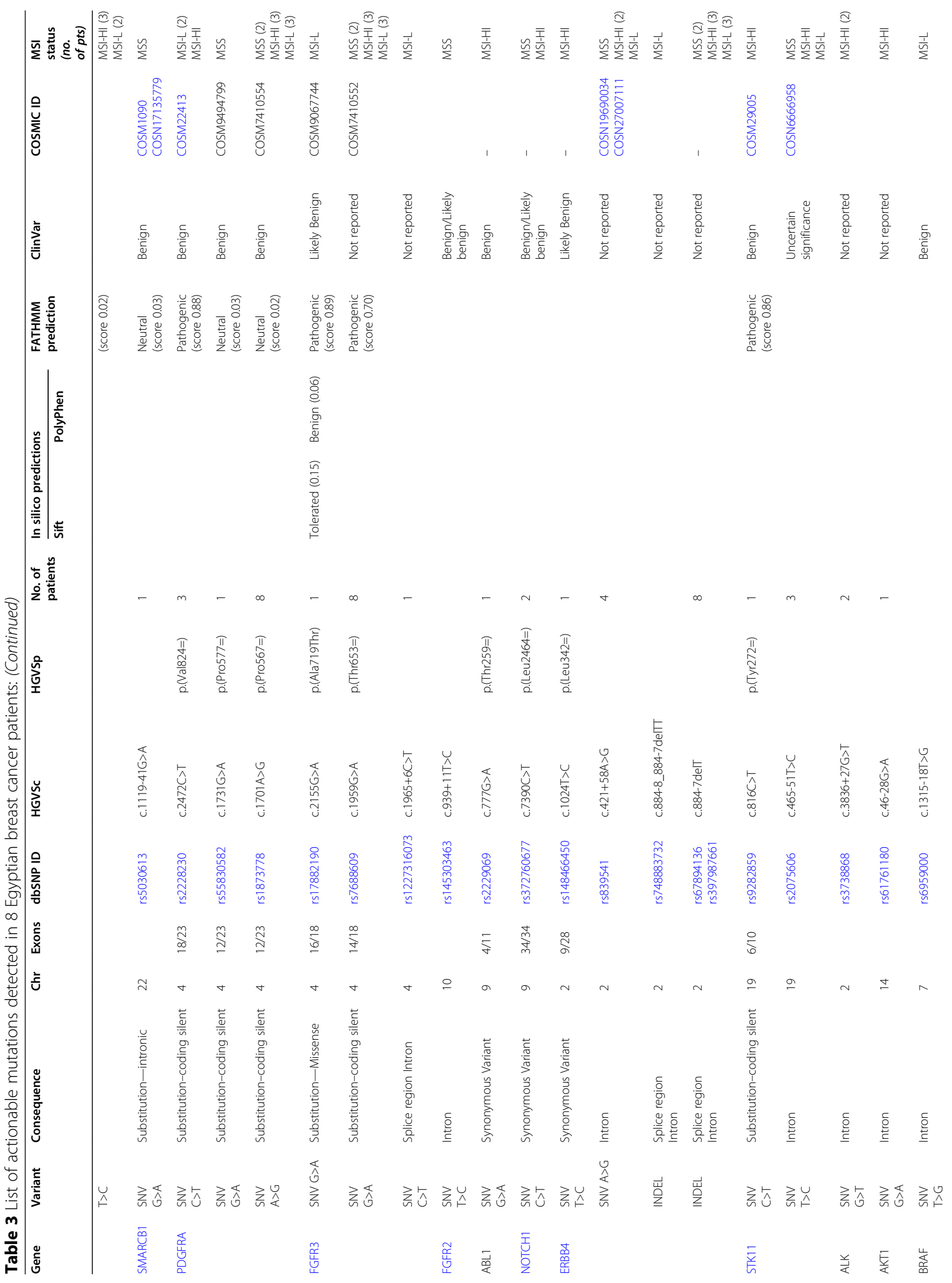




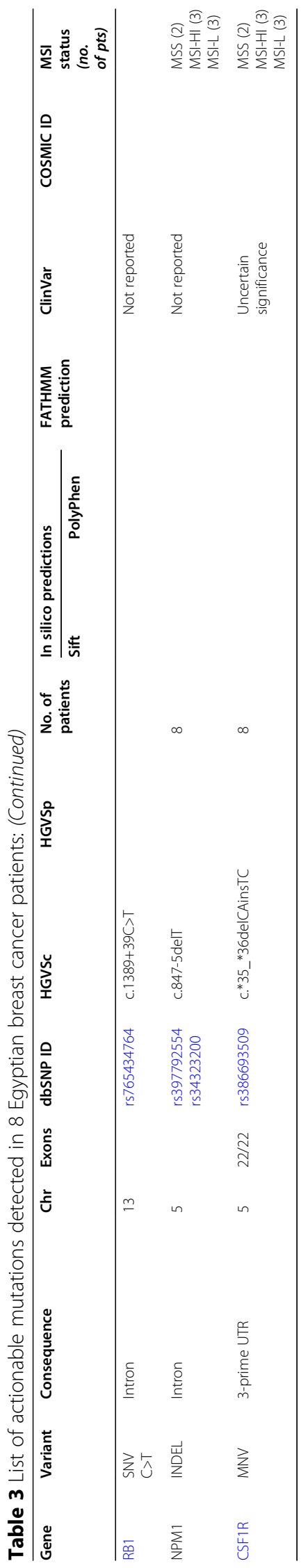




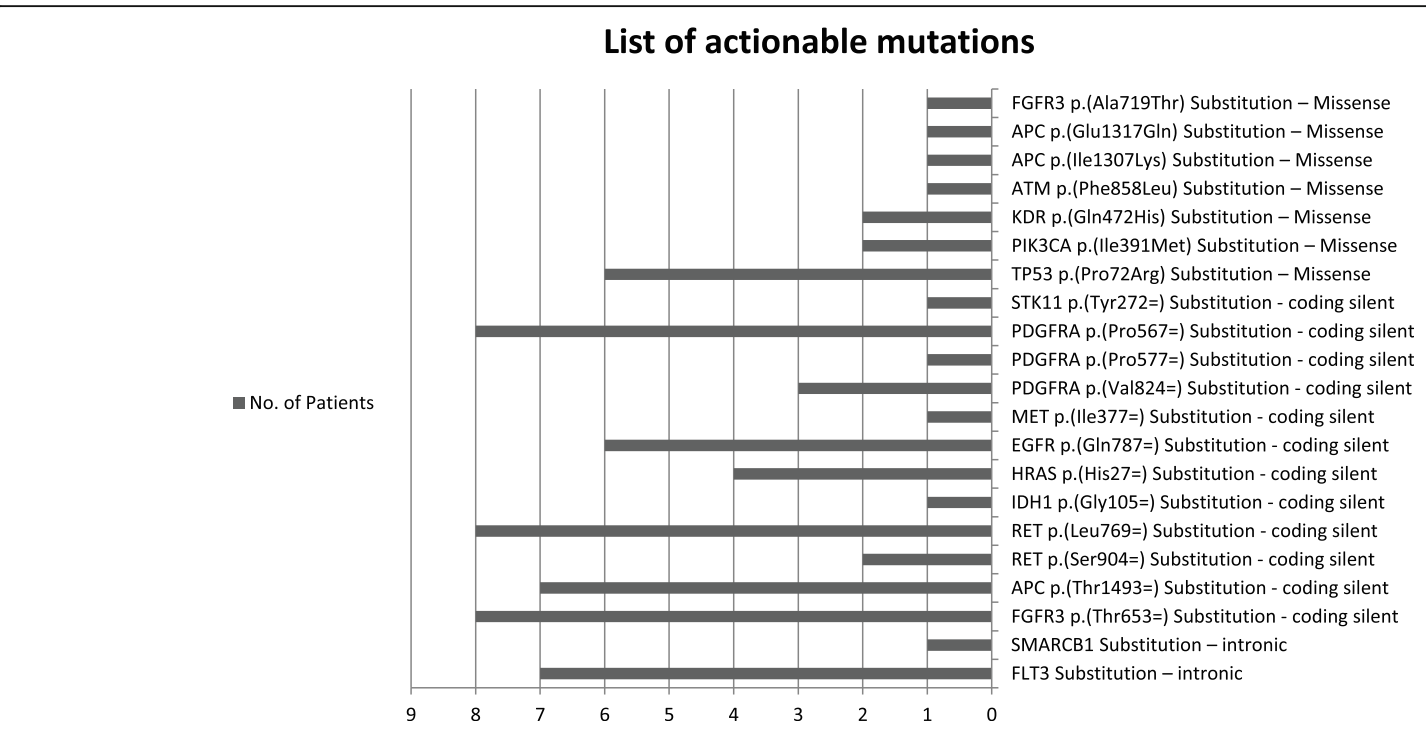

Fig. 1 List of actionable mutations in studied breast cancer patients

Correlation between MSI status and different studied somatic mutations was shown in Fig. 2.

\section{Discussion}

The application of liquid biopsy in solid tumors affords a useful and secure method to identify the existence of actionable driver mutations, to monitor response to therapy, to discover recent recurrence, to aid the radiological imaging in post-treatment surveillance, and to predict cancer therapy outcomes [16]. The crucial intent of precision medicine in cancer patients is to adjust clinical management according to targeted molecular profiling. Next-generation sequencing is increasingly used in identification of somatic mutations in each cancer patient and such information can direct treatment decisions [17]. Here, we identify the activating mutations in liquid biopsy of Egyptian breast cancer patients using targeted next generation sequencing technology. Also, we detect the microsatellite instability (MSI) status using BAT25, BAT26, and NR27 markers which are tested on the Bioanalyzer 2100 system.

TP53 gene is a tumor suppressor gene that control DNA repair and apoptosis mechanisms. TP53 mutation is frequently observed in $\mathrm{BC}$ and it accounts for nearly $30 \%$ of all BC cases. In our study, all TP53 mutations were p.(Pro72Arg) drug response variant and found in 6 out of 8 patients $(75 \%)$. It was described previously that TP53 p.(Pro72Arg) variant denotes BC susceptibility [18]. On the other hand, some reports found that TP53 p.(Pro72Arg) revealed no significant association with BC

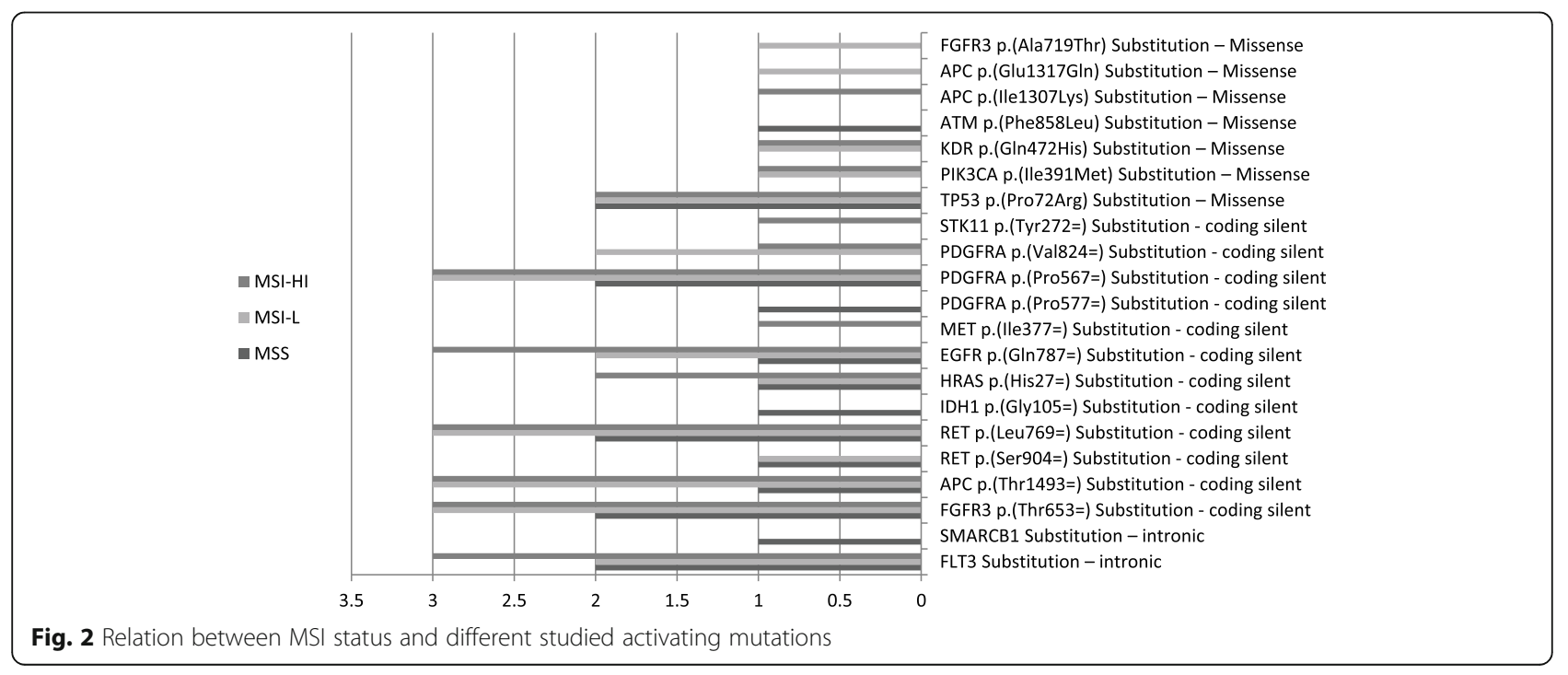


risk $[19,20]$. In addition, it was noted that TP53 mutation with the R72 variant was significantly correlated with poor prognosis in BC females. Therefore, TP53 codon 72 might be a powerful anticipating marker for chemotherapeutic response in $\mathrm{BC}$ [21].

PIK3CA mutations are detected in 30-40\% of BC patients and lead to alpha isoform ( $1110 \alpha)$ of the phosphatidylinositol 3-kinase (PI3K) hyperactivation [22]. We found that 2 out of our 8 patients (25\%) revealed PIK3CA p.(Ile391Met) mutation. These 2 patients were metastatic BC patients, one of them was stage III and the other was stage IV at diagnosis. Nassar et al. 2020 found PIK3CA I391M polymorphism in 7 patients (15.2\%) and revealed that it could be used as BC tumorigenesis marker [23]. Ahmadi et al. 2017 revealed that PIK3CA I391M (rs2230461 A>G) genetic polymorphism is not correlated with breast cancer risk. Yet, he found significant differences in stage III BC patients compared to control group which may be a molecular sign that reveals the PIK3CA rs2230461 can be associated with the starting of breast cancer cells invasion [24]. Finally, in a pre-clinical experiment, trastuzumab efficacy was appraised against many HER2positive cell lines. ZR-75-30 cell line expressing PIK3CA I391M was associated with same sensitivity to trastuzumab in comparison to SK-BR-3 cell line expressing PIK3CA wild-type, at all concentrations tested $(0-10 \mu \mathrm{g} / \mathrm{mL})$ [25].

Kinase insert domain receptor $(K D R)$ gene is a vascular endothelial growth factor receptor 2 (VEGFR2) gene. The PI3K/Pten pathway is one of the downstream signalings affected by KDR activation and most commonly altered in breast cancer [26]. In our study, KDR p.(Gln472His) mutation was found in the same 2 patients who revealed PIK3CA p.(Ile391Met) mutation.

Two patients showed APC substitution-missense mutations, one patient showed p.(Ile1307Lys) variant, and the other patient was p.(Glu1317Gln). APC p.(Ile1307Lys) was reported previously as risk factor for susceptibility to BC [27]. The APC p. Glu1317Gln variant is known as conflicting interpretation of pathogenicity in ClinVar, although it may have pathogenic effect and was detected in one pancreatic cancer patient and one breast cancer patient from different families [28].

Finally, only one patient revealed ATM p.(Phe858Leu) gene mutation. In US, this missense mutation occurred at $\sim 2 \%$ frequency and was associated with a significant increased BC risk [29].

Microsatellite instability (MSI) is remarkably low in $\mathrm{BC}$, in spite of extensive clinical expectations that various patients might be responsive to immune checkpoint inhibitors [30]. However in our study, 2/8 (25\%) patients were MSS, $3 / 8(37.5 \%)$ patients were MSI-L, and $3 / 8$ (37.5\%) patients were MSI-HI. A relatively larger cohort is needed for further and precise analysis of these genetic markers and MSI status in Egyptian BC patients.

\section{Conclusion}

It is essential to use and validate minimally invasive liquid biopsy for activating mutations detection by nextgeneration sequencing especially in patients with inoperable disease or bone metastasis. This work should be extended with larger patient series with comparison of genetic mutations in liquid-based versus tissue-based biopsy and longer follow up period.

\section{Abbreviations \\ APC: APC Regulator of WNT signaling pathway; ATM: Ataxia telangiectasia mutated; BC: Breast cancer; COSMIC: Catalogue of Somatic Mutations in Cancer; ctNA: Circulating tumor nucleic acids; EGFR: Epidermal growth factor receptor; FATHMM: Functional Analysis through Hidden Markov Models (v2.3); FFPE: Formalin-fixed paraffin-embedded; FLT3: Fms-related receptor tyrosine kinase 3; gDNA: Genomic DNA; GRCh37: Genome Reference Consortium Human Build 37; HRAS: HRas Proto-Oncogene, GTPase; HS: High sensitivity; IDC: Invasive duct carcinoma; IDH1: Isocitrate dehydrogenase $(\mathrm{NADP}(+)) 1$; IRB: Institutional Review Board; KDR: Kinase insert domain receptor; MET: MET proto-oncogene, receptor tyrosine kinase; \\ MSI: Microsatellite instability; MSI-HI: Microsatellite instability-high; MSI- L: Microsatellite instability- low; MSS: Microsatellite stable; NCRP: National Cancer Registry Program; NGS: Next-generation sequencing; PDGF RA: Platelet-derived growth factor receptor alpha; \\ PIK3CA: Phosphatidylinositol 4,5-bisphosphate 3-kinase catalytic subunit alpha; Q-score: Quality score; RET: Ret proto-oncogene; SMARCB1: SWI/SNF Related, Matrix Associated, Actin Dependent Regulator of \\ ChromatinSubfamily B, member 1; STK11: Serine/threonine kinase 11; TP53: Tumor protein TP53; VAF: Variant allele frequency; VCF: Variant Call Format; VEGFR2: Vascular endothelial growth factor receptor 2}

\section{Acknowledgements}

We deeply acknowledge the scientific and technical support offered by Prof. Dr. Wafaa El Metinawy, Prof. Dr. Hamdy Abdel Azim and Prof. Dr. Emad Hamada. We are also thankful for our molecular lab team; Nashwa Medhat and Basant Nagdy .

\section{Authors' contributions}

NK analyzed and interpreted the patient data as well as bioinformatics. HK was responsible for data analysis, bioinformatics, and was a major contributor in writing the manuscript. LK was responsible for demographic and clinical data collection. $\mathrm{MH}$ analyzed and interpreted the clinical data. All authors read and approved the final .manuscript

\section{Funding}

This study was funded by Kasr Al Ainy Clinical Oncology dep., Cairo University which provides us with the needed kits. Needed laboratory analysis was performed in molecular Lab. of Kasr Al-Ainy Clinical Oncology dep. with the availability of all equipments needed in our study.

\section{Availability of data and materials}

The datasets used and/or analyzed during the current study are available from the corresponding author on reasonable request.

\section{Declarations}

Ethics approval and consent to participate

This study was approved by Kasr Al Ainy Clinical Oncology department Institutional Review Board (IRB)-11-2019. Written informed consent was obtained from all individual participants included in the study. All procedures performed in the study involving human participants were in accordance with the ethical standards of the institutional research committee and with the 1964 Helsinki declaration and its later amendments (GCP guidelines) or comparable ethical standards.

Consent for publication

The consent to publish had been taken from each participant in this work. 


\section{Competing interests}

The authors declare that they have no competing interests.

\section{Author details}

${ }^{1}$ Clinical and Chemical Pathology Department, Kasr Al Ainy Centre of Clinical Oncology \& Nuclear Medicine, School of Medicine, Cairo University, Cairo, Egypt. ${ }^{2}$ Clinical Oncology Department, School of Medicine, Cairo University, Cairo, Egypt.

Received: 20 January 2021 Accepted: 9 April 2021

Published online: 17 April 2021

\section{References}

1. Talima S, Kassem H, Kassem N. Chemotherapy and targeted therapy for breast cancer patients with hepatitis C virus infection. Breast Cancer. 2019; 26(2):154-63. https://doi.org/10.1007/s12282-018-0904-2.

2. Kassem NM, Makar WS, Kassem HA, Talima S, Tarek M, Hesham H, et al. Circulating miR-34a and miR-125b as promising non invasive biomarkers in Egyptian locally advanced breast cancer patients. Asian Pac J Cancer Prev. 2019:20(9):2749-55. https://doi.org/10.31557/APJCP.2019.20.9.2749.

3. Savci-Heijink CD, Halfwerk H, Hooijer GK, et al. Retrospective analysis of metastatic behaviour of breast cancer subtypes. Breast Cancer Res Treat. 2015;150(3):547-57. https://doi.org/10.1007/s10549-015-3352-0.

4. Kono M, Fujii T, Matsuda N, et al. Somatic mutations, clinicopathologic characteristics, and survival in patients with untreated breast cancer with bone-only and non-bone sites of first metastasis. J Cancer. 2018;9(19):36406Published 2018 Sep 8. https://doi.org/10.7150/jca.26825.

5. Vogelstein B, Papadopoulos N, Velculescu VE, Zhou S, Diaz LA, Kinzler KW. Cancer genome landscapes. Science. 2013;339(6127):1546-58. https://doi. org/10.1126/science.1235122

6. Bamshad MJ, Ng SB, Bigham AW, Tabor HK, Emond MJ, Nickerson DA, et al. Exome sequencing as a tool for mendelian disease gene discovery. Nat Rev Genet. 2011;12(11):745-55. https://doi.org/10.1038/nrg3031.

7. Zhang P, Lehmann BD, Shyr Y, Guo Y. The utilization of formalin fixedparaffin-embedded specimens in high throughput genomic studies. Int J Genom. 2017;2017:1926304. https://doi.org/10.1155/2017/1926304 PMID: 28246590.

8. Do H, Dobrovic A. Sequence artifacts in DNA from formalin-fixed tissues: causes and strategies for minimization. Clin Chem. 2015;61(1):64-71. https:// doi.org/10.1373/clinchem.2014.223040 PMID: 25421801.

9. Wu SG, Shih JY. Management of acquired resistance to EGFR TKI-targeted therapy in advanced nonsmall cell lung cancer. Mol Cancer. 2018;17(1):38. https://doi.org/10.1186/s12943-018-0777-1 PMID: 29455650.

10. Stroun M, Anker P, Maurice P, Lyautey J, Lederrey C, Beljanski M. Neoplastic characteristics of the DNA found in the plasma of cancer patients. Oncology. 1989;46(5):318-22. https://doi.org/10.1159/000226740 PMID: 2779946.

11. Dominguez-Vigil IG, Moreno-Martinez AK, Wang JY, Roehrl MHA, BarreraSaldana HA. The dawn of the liquid biopsy in the fight against cancer Oncotarget. 2018;9(2):2912-22. https://doi.org/10.18632/oncotarget.23131 PMID: 29416824

12. Mathai RA, Vidya RVS, Reddy BS, Thomas L, Udupa K, Kolesar J, Rao M Potential Utility of Liquid Biopsy as a Diagnostic and Prognostic Tool for the Assessment of Solid Tumors: Implications in the Precision Oncology. J Clin Med. 2019(3):373. https://doi.org/10.3390/jcm8030373.

13. Heitzer E, Haque IS, Roberts CES, Speicher MR. Current and future perspectives of liquid biopsies in genomics-driven oncology. Nat Rev Genet. 2019;20(2):71-88. https://doi.org/10.1038/s41576-018-0071-5 PMID: 30410101.

14. Catalogue of Somatic Mutations in Cancer - Home Page. http://cancer.sa nger.ac.uk/cosmic. Accessed 25 Oct 2017.

15. Kassem NM, Emera G, Kassem HA, et al. Clinicopathological features of Egyptian colorectal cancer patients regarding somatic genetic mutations especially in KRAS gene and microsatellite instability status: a pilot study. Egypt J Med Hum Genet. 2019;20(1):20. https://doi.org/10.1186/s43042-0190028-z.

16. Wan JCM, Massie C, Garcia-Corbacho J, Mouliere F, Brenton JD, Caldas C, et al. Liquid biopsies come of age: towards implementation of circulating tumour DNA. Nat Rev Cancer. 2017;17(4):223-38. https://doi.org/10.1038/ nrc.2017.7.
17. Kassem N, Sharaf S, Abdel Aziz A, Mohsen M, Kassem H, El Khateeb S, et al. Towards validation of targeted next-generation sequencing on formalin fixed paraffin embedded colorectal cancer tissues in Egyptian population: a pilot study with feasibility and challenges. Int J Cancer Tremnt. 2018;1(1):20-9.

18. Zhang Z, Wang M, Wu D, Tong N, Tian Y. P53 codon 72 polymorphism contributes to breast cancer risk: a meta-analysis based on 39 case-control studies. Breast Cancer Res Treat. 2010;120:509-17.

19. Ma Y, Yang J, Liu Z, Zhang P, Yang Z, Wang Y, et al. No significant association between the TP53 codon 72 polymorphism and breast cancer risk: a metaanalysis of 21 studies involving 24,063 subjects. Breast Cancer Res Treat. 2011;125(1):201-5. https://doi.org/10.1007/s10549-010-0920-1.

20. Cheng H, Biao Ma B, Jiang R, Wang W, Guo H, et al. Individual and combined effects of MDM2 SNP309 and TP53 Arg72Pro on breast cancer risk: an updated meta-analysis. Mol Biol Rep. 2012;39(9):9265-74. https://doi. org/10.1007/s11033-012-1800-z.

21. Basu S, Gnanapradeepan K, Barnoud T, Kung CP, Tavecchio M, Scott J, et al. Mutant p53 controls tumor metabolism and metastasis by regulating PGC1a. Genes Dev. 2018;32(3-4):230-43. https://doi.org/10.1101/gad.309062.117.

22. Mollon L, Aguilar A, Anderson E, et al. Abstract 1207: a systematic literature review of the prevalence of PIK3CA mutations and mutation hotspots in HR+/HER2- metastatic breast cancer. Cancer Res. 2018;78:1207.

23. Nassar A, Abouelhoda M, Mansour O, Loutfy SA, Hafez MM, Gomaa M, et al. Targeted next generation sequencing identifies somatic mutations in a cohort of Egyptian breast cancer patients. J Adv Res. 2020;24:149-57. https://doi.org/10.1016/j.jare.2020.04.001.

24. Ahmadi S, Ramezani S, Ghafouri H, Hosseini SM, Najafi A, Keihan AH. Association between the PIK3CA Ile391Met polymorphism and the risk of breast cancer in an Iranian population. J Appl Biotechnol Rep. 2018:5(1):812. https://doi.org/10.29252/JABR.01.01.02.

25. Baselga J, Lewis Phillips GD, Verma S, Ro J, Huober J, Guardino AE, et al. Relationship between tumor biomarkers and efficacy in EMILIA, a phase III study of trastuzumab emtansine in HER2-positive metastatic breast cancer. Clin Cancer Res. 2016;22(15):3755-63. https://doi.org/10.1158/1078-0432. CCR-15-2499 Epub 2016 Feb 26. Erratum in: Clin Cancer Res. 2018 Nov 1; 24(21):5486. PMID: 26920887; PMCID: PMC5485412.

26. Dai $X$, Mei $Y$, Chen $X$, Cai D. ANLN and KDR are jointly prognostic of breast cancer survival and can be modulated for triple negative breast cancer control. Front Genet. 2019;10:790. https://doi.org/10.3389/fgene.2019.00790.

27. Petersen GM, Parmigiani G, Thomas D. Missense mutations in disease genes: a Bayesian approach to evaluate causality. Am J Hum Genet. 1998;62(6): 1516-24. https://doi.org/10.1086/301871.

28. Earl J, Galindo-Pumarinoa C, Encinasa J, Barretoa E, Castilloa ME, Pachona V, et al. A comprehensive analysis of candidate genes in familial pancreatic cancer families reveals a high frequency of potentially pathogenic germline variants. EBioMedicine. 2020;53:102675. https://doi.org/10.1016/j.ebiom.202 0.102675 .

29. Stredrick DL, Garcia-Closas M, Pineda MA, Bhatti P, Alexander BH, Doody MM, et al. The ATM missense mutation p.Ser49Cys (c.146C $>\mathrm{G}$ ) and the risk of breast cancer. Hum Mutat. 2006;27(6):538-44. https://doi.org/10.1002/ humu.20323.

30. Horimoto $Y$, Thinzar Hlaing M, Saeki H, Kitano S, Nakai K, Sasaki R, et al. Microsatellite instability and mismatch repair protein expressions in lymphocyte-predominant breast cancer. Cancer Sci. 2020;111(7):2647-54. https://doi.org/10.1111/cas.14500.

\section{Publisher's Note}

Springer Nature remains neutral with regard to jurisdictional claims in published maps and institutional affiliations. 